\title{
Correction to: Machine learning based on clinico-biological features integrated 18F-FDG PET/CT radiomics for distinguishing squamous cell carcinoma from adenocarcinoma of lung
}

\author{
Caiyue Ren ${ }^{1,2} \cdot$ Jianping Zhang ${ }^{3,4,5,6} \cdot$ Ming $\mathbf{i}^{3,4,5,6} \cdot$ Jiangang Zhang ${ }^{1,2} \cdot$ Yingjian Zhang ${ }^{2,3,4,5,6} \cdot$ Shaoli Song ${ }^{1,2,4,5,6}$. \\ Yun Sun ${ }^{1,3,7}$ • Jingyi Cheng ${ }^{2,3,4,5,6}$
}

Published online: 2 February 2021

(C) Springer-Verlag GmbH Germany, part of Springer Nature 2021

\section{Correction to: Eur J Nucl Med Mol Imaging} https://doi.org/10.1007/s00259-020-05065-6

There was a mistake in one of the article notes found in the original article.

"These authors contributed equally to this work."

should have read as

"Ren Caiyue and Zhang Jianping contributed equally in this work."

The original article has been corrected.

Publisher's note Springer Nature remains neutral with regard to jurisdictional claims in published maps and institutional affiliations.

This article is part of the Topical Collection on Erratum

The online version of the original article can be found at https://doi.org/ $10.1007 / \mathrm{s} 00259-020-05065-6$

Yun Sun

yun.sun@sphic.org.cn

Caiyue Ren

caiyue.ren@sphic.org.cn

Jianping Zhang

zhangjianpin82@126.com

Ming Qi

qiming531@hotmail.com

Jiangang Zhang

jiangang.zhang@sphic.org.cn

Yingjian Zhang

yingjian.zhang@sphic.org.cn

Shaoli Song

shaoli_song@yeah.net

Jingyi Cheng

jcheng13@ fudan.edu.cn
Department of Nuclear Medicine, Shanghai Proton and Heavy Ion Center, Shanghai 201315, China

2 Shanghai Engineering Research Center of Proton and Heavy Ion Radiation Therapy, Shanghai, China

3 Department of Nuclear Medicine, Shanghai Proton and Heavy Ion Center, Fudan University Cancer Hospital, Shanghai 201321, China

4 Department of Oncology, Shanghai Medical College, Fudan University, Shanghai 200032, China

5 Center for Biomedical Imaging, Fudan University, Shanghai 200032, China

6 Shanghai Engineering Research Center for Molecular Imaging Probes, Shanghai 200032, China

7 Department of Research and Development, Shanghai Proton and Heavy Ion Center, Shanghai 201321, China 